\title{
1.5D BISON Simulation Capability CASL FY17 Letter Report
}

J. D. Hales, S. R. Novascone, B. W. Spencer, H. Chen, S. A. Pitts, A. Casagranda

January 2017

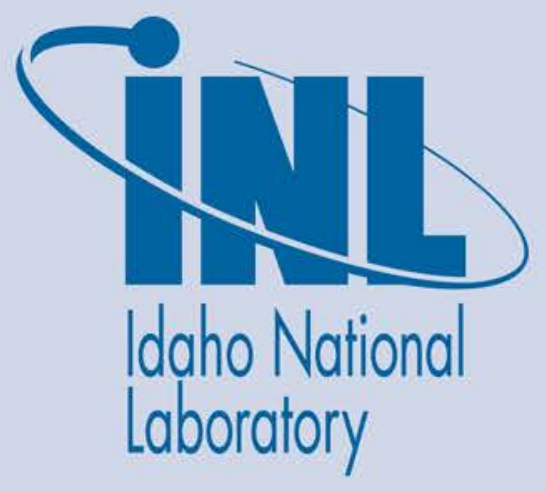

The INL is a U.S. Department of Energy National Laboratory operated by Battelle Energy Alliance 
INL/EXT-16-40785

\subsection{BISON Simulation Capability CASL FY17 Letter Report}

J. D. Hales, S. R. Novascone, B. W. Spencer, H. Chen, S. A. Pitts, A. Casagranda

January 2017

Idaho National Laboratory

Idaho Falls, Idaho 83415

http://www.inl.gov

Prepared for the

U.S. Department of Energy

Assistant Secretary for __, OR Office of

Under DOE Idaho Operations Office

Contract DE-AC07-05ID14517 


\title{
1.5D BISON Simulation Capability CASL FY17 Letter Report
}

\author{
J. D. Hales, S. R. Novascone, B. W. Spencer, H. Chen, S. A. Pitts, A. Casagranda \\ Fuels Modeling and Simulation \\ Idaho National Laboratory \\ P.O. Box 1625 \\ Idaho Falls, ID 83415-3840 \\ January 11, 2017
}

\section{Introduction}

This milestone report documents the recently-developed capability to model light water reactor (LWR) fuel rods using a 1.5 dimensional representation in BISON. As the application of BISON expands, the design requirements evolve. The initial emphasis of BISON development focused on multi-dimensional/multi-physics fuel performance simulation. BISON was originally developed to solve 2D axisymmetric and 3D models, which permits it to model a wide variety of fuel forms and local phenomena that cannot be represented using lower dimensionality models.

While a higher dimensionality capability is very powerful, there are many fuel modeling problems that can be adequately represented using models with lower dimensionality. Modeling a problem with with higher dimensionality than is mandated by the physics and geometry provides an accurate solution, but requires more complexity and computational cost than is necessary. The MOOSE framework that BISON is built on provides the flexibility to use the same physics models (with some relatively minor modifications in some cases) in $1 \mathrm{D}, 2 \mathrm{D}$, or $3 \mathrm{D}$, so that the appropriate representation for a given problem of interest can be used.

The use of 1D models in BISON is not without precedent - a 1D spherically symmetric capability was developed and successfully applied to model TRISO fuel in BISON, as documented in [1].

The primary motivation for the effort documented here is that the CASL program has demonstrated the need for a fast-running, simplified version of BISON for use in large multi-physics simulations involving hundreds of individual fuel rods in VERA. The 2D axisymmetric models currently being used for this purpose successfully model the physics of interest, but are unnecessarily complex and computationally intensive to represent the phenomenon of interest, which is primarily radial heat transfer. This document describes the development of the 1.5D LWR capability, demonstrates this capability on an LWR rodlet, and identifies needs for future development.

\section{Development}

The term "1.5D" is used here to describe a model of a cylindrical fuel geometry that is a collection of coupled 1D simulations used to represent the behavior of the fuel at given axial positions along the rod. The fuel rod is divided into several axial slices, and a 1D model of the physics (energy conservation, stress divergence, thermal and mechanical contact) is solved on each slice. An illustration of these 1D axial slices is shown in Figure 1.

The following equations for energy conservation and stress divergence, respectively, are solved simultaneously using 1D axisymmetric models for each axial slice:

$$
\begin{gathered}
\rho C_{p} \frac{\partial T}{\partial t}-\nabla \cdot k \nabla T-q=0 \\
\nabla \cdot \sigma=0
\end{gathered}
$$


where $\rho$ is the mass density, $C_{p}$ is the specific heat, $T$ is the temperature, $k$ is the thermal conductivity, $q$ is the volumetric heat generation rate, and $\sigma$ is the stress. Each slice represents the behavior of a finite axial segment of the rod, over which the behavior is assumed to be uniform.

Because a given 1D model is only applicable for a finite length of the fuel rod, and in general the behavior of a rod varies significantly over its length, an ability to represent axial variations is necessary. This is done by adding features that permit the rod to be modeled using a collection of $1 \mathrm{D}$ models, thus making it a "1.5D" model. To accomplish this, modified versions of a number of code modules in BISON that compute integral quantities were created specifically for 1.5D models. These code modules are aware the domain is composed of a set of $1 \mathrm{D}$ models with finite axial length, and perform the integrals in an appropriate manner. Examples of these modules include those that calculate plenum volume, fission gas released, and heat flux. Additionally, specialized code to compute the axial pressure applied from the coolant and plenum pressure was developed.

A mesh for a 1.5D LWR fuel model consists of rows of $1 \mathrm{D}$ elements at each elevation to represent the fuel and cladding, and a row of elements to represent the cladding in the plenum region, as illustrated in Figure 1. This mesh can be created externally to BISON, but to facilitate the setup of such models, a built-in 1.5D mesh generator was developed, and it is expected that this will be the primary way that such models are set up. This mesh generator requires basic information about the fuel and cladding geometry and the desired discretization.

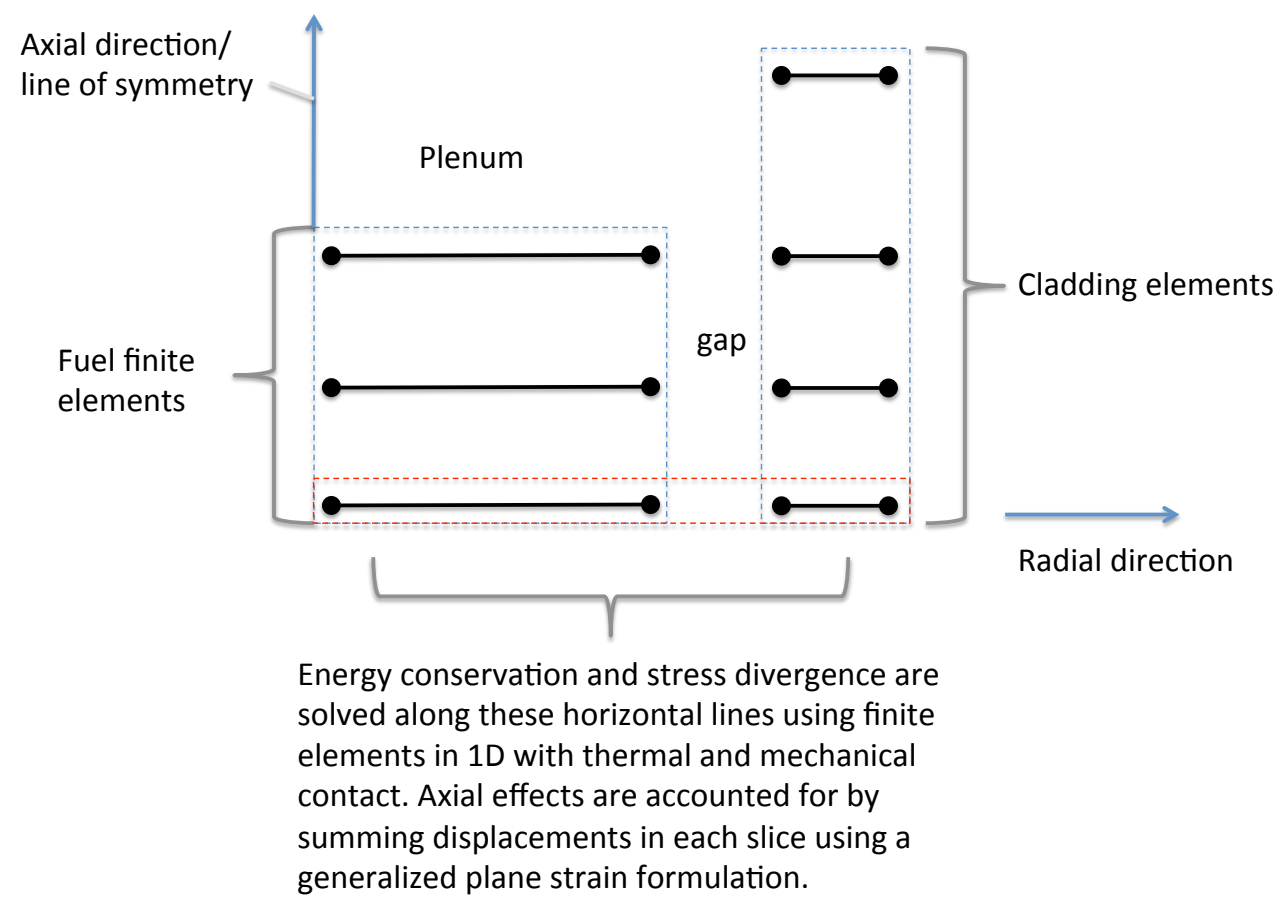

Figure 1: $1.5 \mathrm{D}$ problem and mesh illustration

An important considering in modeling a finite axial segment of the rod in 1D is that a generalized plane strain condition must be imposed on the mechanical response in the axial direction. In solid mechanics, plane strain is the condition, or assumption, that out-of-plane strains are zero. In the case of a fuel rod, the axial strains for a given section of the rod must be uniform (so that planar sections remain planar), but it must also in general be nonzero to allow for the effects of thermal expansion and other phenomena leading to volume change.

A generalized plane strain capability solves for the out-of-plane strain that satisfies equilibrium. Such a capability 
was previously developed for 2D Cartesian models using a field variable describing the out-of-plane strain and a constraint that forced that variable to be equal everywhere in an element block. Motivated by the current 1.5D development efforts, an improved version of this has been developed that uses a global scalar variable to represent the out-of-plane strain for an element block, which significantly reduces the size of the system of equations and improves solution robustness. This new capability is applicable to both $1 \mathrm{D}$ and $2 \mathrm{D}$ models. In $1.5 \mathrm{D}$ LWR fuel rods, an independent generalized plane strain condition is imposed on the fuel and cladding at every elevation.

\section{Demonstration}

The BISON source code repository at hpcgitlab.inl.gov contains an example BISON LWR problem, which is a 10-pellet fuel rod modeled as 2DRZ (axisymmetric) with all of the current material models and recommended options and serves as a starting point for most BISON simulations. An analogous input file was created for the 1.5D case. This model is currently located in the examples/tensor_mechanics/1.5D_rodlet_10pellets directory of the BISON repository.

Comparisons between results from this 1.5D model and the equivalent 2DRZ model were made. Comparisons of a few representative quantities of interest are shown in this section. Figure 2 shows temperature contours at the end of the simulation for the $1.5 \mathrm{D}$ and $2 \mathrm{D}$ models, which agree quite well.

Time histories of a number of quantities of interest also show very good agreement between the 1.5D and 2D models. Time histories of the temperature in the fuel and cladding are shown in Figure 3 and 4 respectively. Similarly, von Mises stress in the cladding is shown in Figure 5 and the radial displacement profiles along the fuel and cladding are shown at the end of the simulation in Figure 6.

Finally, Figure 7 shows the simulation run time required for the two models as a function of the analysis time. The overall run time for the $1.5 \mathrm{D}$ model is over 10x faster than its $2 \mathrm{D}$ equivalent, which is encouraging. For full length rods, 2D axisymmetric models often require a much larger number of elements in the axial direction than this model does. Full length rods can still be represented with a relatively small number of axial segments in $1.5 \mathrm{D}$, however, so a much greater speedup relative to the 2D models than is shown here is expected in those cases.

\section{Summary and Future Work}

This report documents the development and initial demonstration of a capability to model LWR fuel rods using a 1.5D representation. The results presented here show very good agreement between $1.5 \mathrm{D}$ and $2 \mathrm{D}$ axisymmetric models of a short LWR fuel rod. The 1.5D model runs 10x faster than the 2D model, and much greater speedups are expected for full-length rod models.

The 1.5D capability has been incorporated into the current BISON development code branch, and is available for testing. The following tasks remain to prepare this capability for widespread use:

- Usability Improvements: To use this capability, a user must currently define a set of objects in the input file corresponding to the fuel and cladding at each individual elevation of the fuel rod. This makes the setup and usage of this capability somewhat unwieldy and error-prone. Work is planned in the near future to develop code that will automatically generate these objects, greatly improving the usability of this capability.

- Validation: The current capability has only been benchmarked against a BISON simulation of a short 2DRZ fuel rod model. A subset of the BISON LWR validation cases [2] are to be simulated in $1.5 \mathrm{D}$ early this year to further assess this new capability.

\section{References}

[1] J. D. Hales, R. L. Williamson, S. R. Novascone, D. M. Perez, B. W. Spencer, and G. Pastore. Multidimensional multiphysics simulation of TRISO particle fuel. J. Nucl. Mater., 443:531-543, 2013.

[2] R.L. Williamson, K.A. Gamble, D.M. Perez, S.R. Novascone, G. Pastore, R.J. Gardner, J.D. Hales, W. Liu, and A. Mai. Validating the BISON fuel performance code to integral LWR experiments. Nuclear Engineering and Design, 301:232 - 244, 2016. 


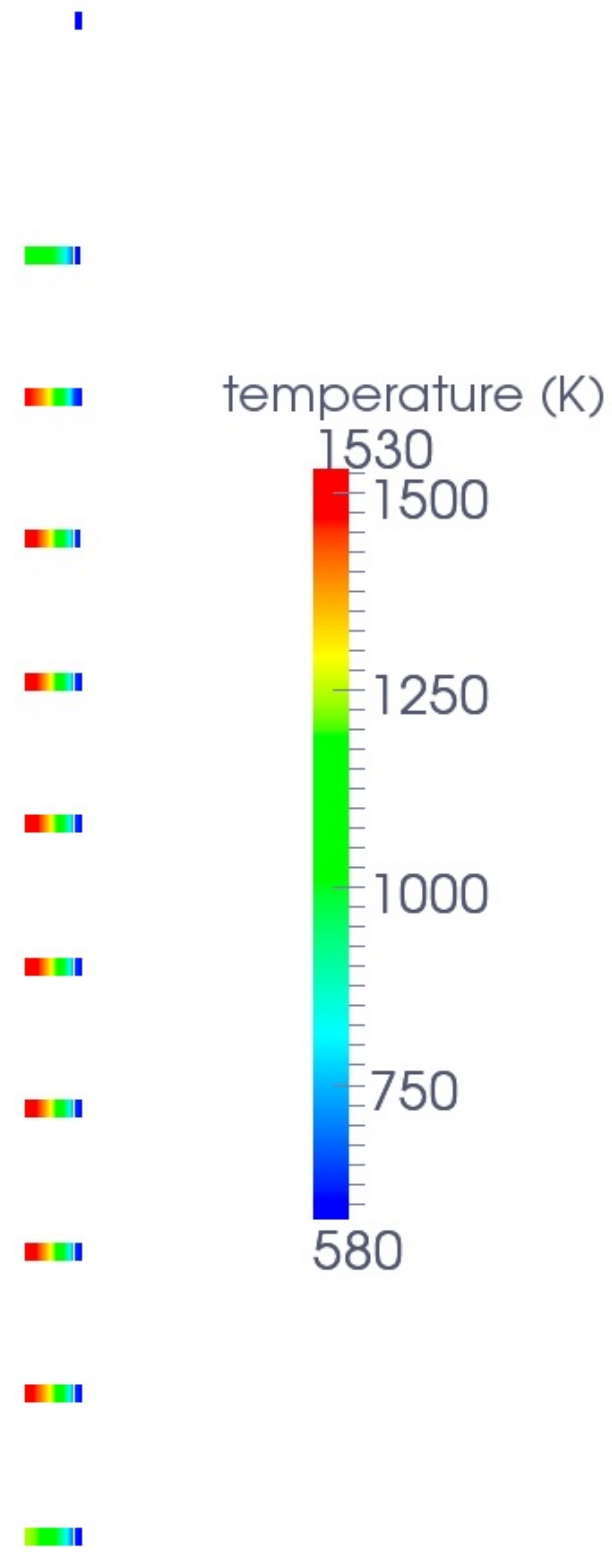

(a) $1.5 \mathrm{D}$ temperature $(\mathrm{K})$ 1515

1500

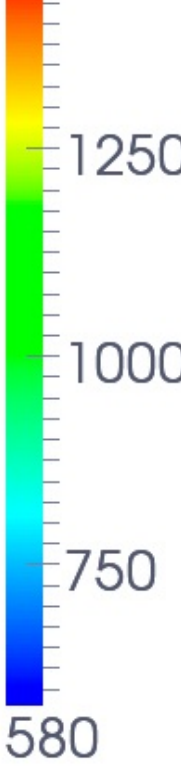

(b) $2 \mathrm{D}$

Figure 2: 1.5D (left) and 2D (right) temperature contour results. Thick lines represent the 1D mesh of the fuel and cladding at each elevation in the $1.5 \mathrm{D}$ model. 


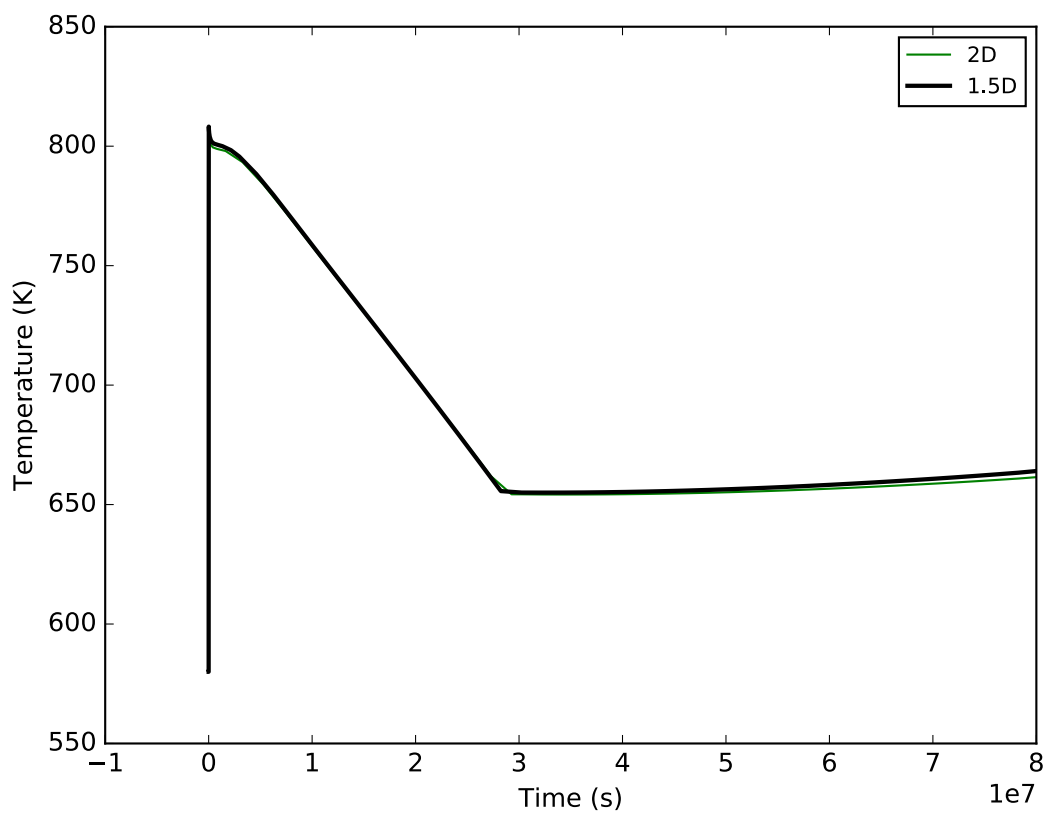

Figure 3: Temperature at fuel surface/axial midpoint vs. time

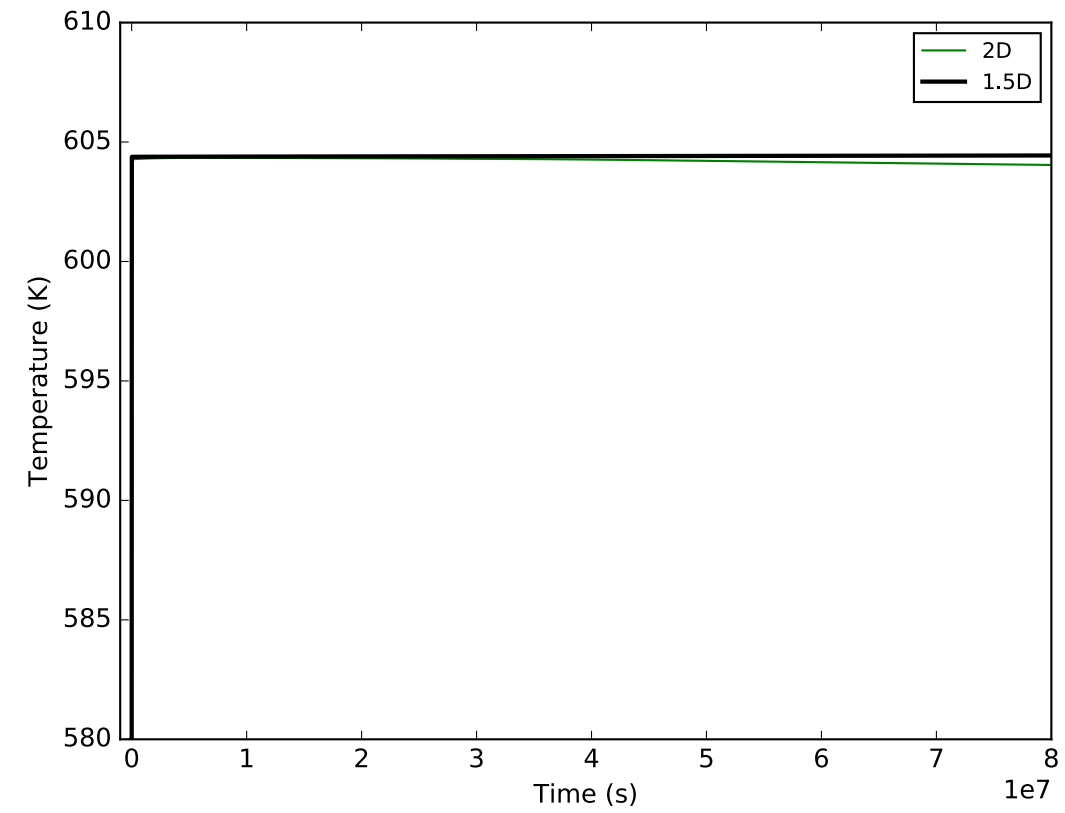

Figure 4: Temperature at cladding surface/axial midpoint vs. time 


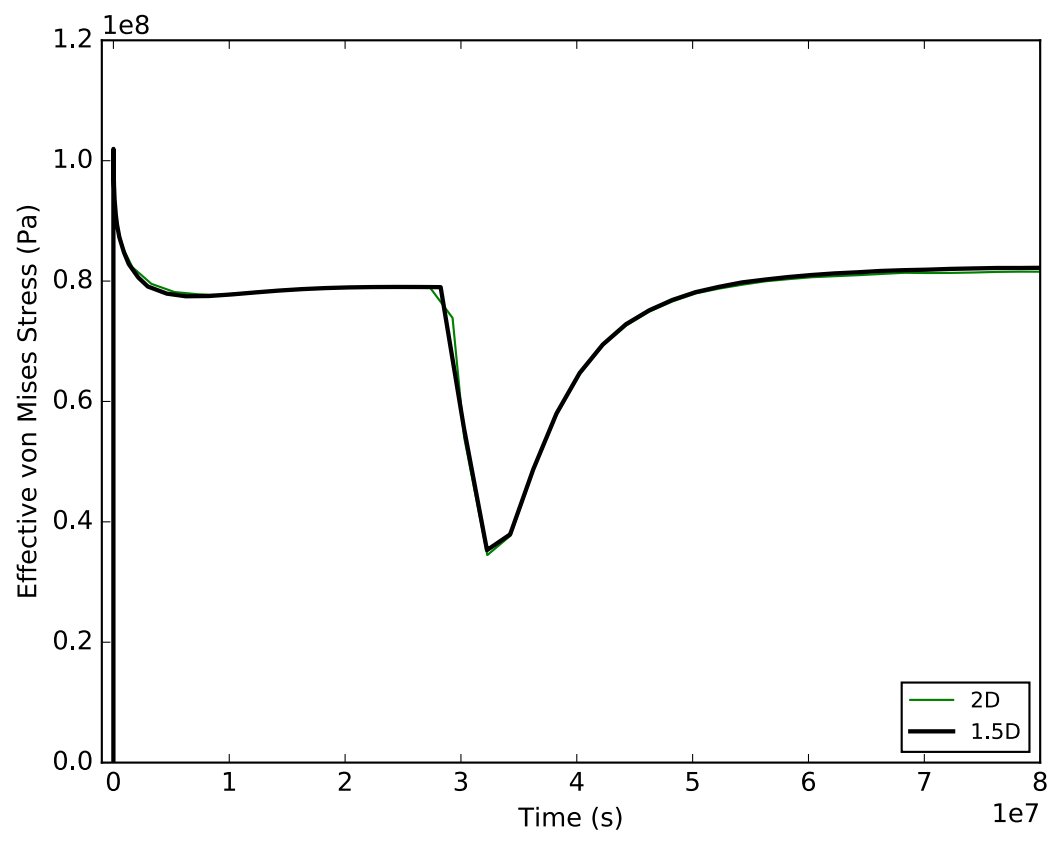

Figure 5: Stress at cladding inner surface/axial midpoint vs. time

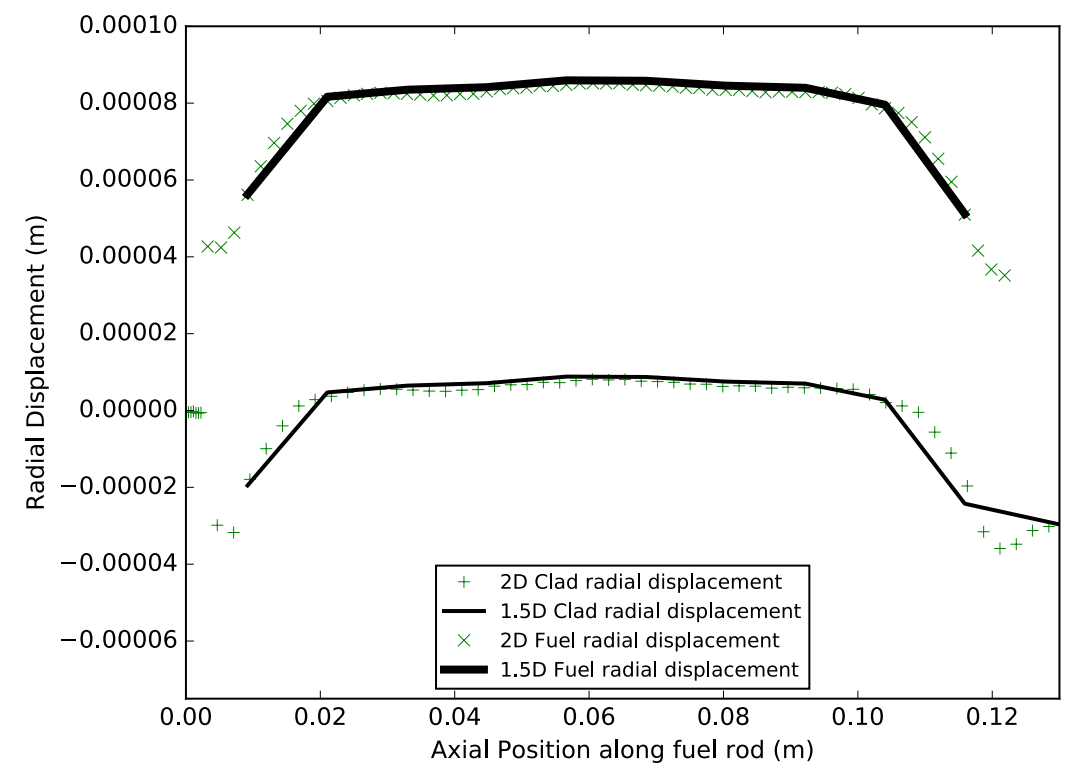

Figure 6: Radial displacement along the vertical axis in fuel and cladding at the end of the simulation. 


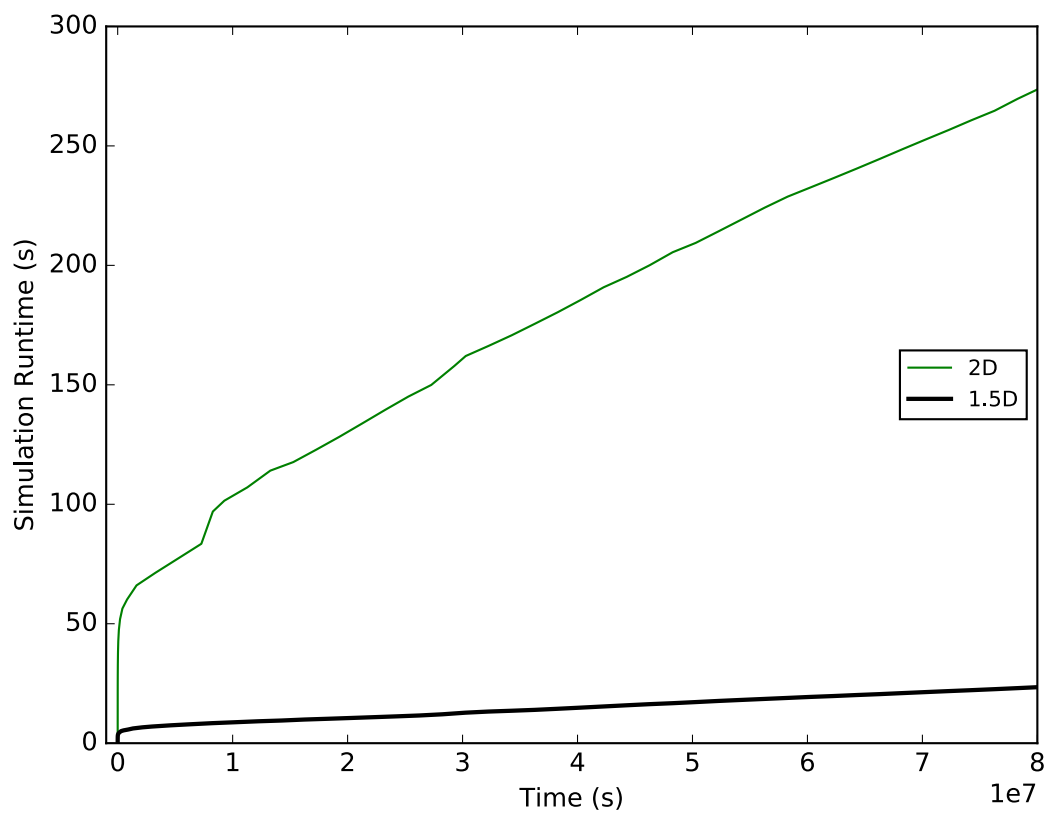

Figure 7: Run time. 